\title{
How does individual variation in sociality influence fitness
}

$5 \quad$ Anne C. Sabol ${ }^{a,{ }^{*}}$, Connor T. Lambert ${ }^{b}$, Brian Keane $^{b}$, Nancy G. Solomon $^{\mathrm{b}},{ }^{\text {Ben Dantzer }}{ }^{\mathrm{a}, \mathrm{c}}$

10 * Correspondence:

11 Anne Sabol

$1211200 \mathrm{SW} 8^{\text {th }}$ St.

13 Miami, FL 33199

14 acsabol72@gmail.com

$15 \quad$ (937) 243-8767

16

17 Declarations of interest: none 


\section{Abstract}

20 Comparative studies aid in our understanding of specific conditions favoring the initial evolution

21 of different types of social behaviors, yet there is much unexplained intraspecific variation in the

22 expression of social behavior that comparative studies have not yet addressed. The proximate

23 causes of this individual variation in social behavior within a species have been examined in

24 some species but its fitness consequences have been less frequently investigated. In this study,

25 we quantified the fitness consequences of variation in the sociality of prairie voles (Microtus

26 ochrogaster). We characterized sociality of voles in semi-natural enclosures using an automated

27 behavioral tracking system paired with social network analyses to quantify the degree of spatial

28 and temporal co-occurrence of different voles. We then assessed the relationship between

29 sociality with mating success (number of different conspecifics with which an individual

30 produced offspring) and reproductive success (total number of offspring surviving to first

31 capture). We measured the number of social connections each individual had with all voles and

32 only with opposite-sex voles by calculating unweighted degree through social network analyses.

33 Both female and male voles varied in the number of social connections they had with all

34 conspecifics and with opposite-sex conspecifics. Voles with an intermediate number of social

35 connections with voles of both sexes had higher mating success overall. In our analyses that

36 considered all social connections with voles of both sexes, voles with an intermediate number of

37 social connections produced more offspring. Males with a very high or low number of social

38 connections also had the lowest average body mass. Overall, our results suggest some limit on

39 the fitness benefits of sociality. Although there was substantial individual-variation in our

40 measure of vole social behavior, intermediate levels of social connections may be most

41 favorable.

42

\section{Keywords}

44 Prairie vole, Social behavior, Social network analyses 


\section{Introduction}

Sociality comes in many forms across a diversity of taxa, ranging from loose, temporary associations during breeding to long-term group associations whose members exhibit complex social behaviors such as cooperative hunting or alloparental care. Different forms of sociality are

49 thought to provide a variety of fitness benefits, including shared resources, reduced predation, 50 communication, or better access to mates (Eisenberg et al., 1972; Alexander, 1974; van Schaik

51 1983; Emlen, 1984; Krause and Ruxton, 2002, Silk, 2007). Sociality may also come with costs

52 associated with group-living, including increased disease transmission (Ewald, 1994; Langwig et

53 al., 2012; Kappeler et al., 2015), parasite load (Côté, and Poulinb, 1995; Whiteman and Parker,

54 2004), physiological stress (Creel et al., 2013), reproductive interference by other group

55 members (Clutton-Brock et al., 1998; Lukas and Huchard, 2014), as well as the time and energy

56 costs devoted to developing and maintaining social connections that could otherwise be

57 devoted towards activities directly related to individual survival or reproduction such as foraging

58 or parental care. Given that these fitness benefits and costs of sociality may vary in direction or

59 magnitude due to ecological circumstances such as food abundance, weather patterns, or

60 temporal variability in these factors (Emlen, 1994; Hatchwell and Komdeur, 2000; Shuster and

61 Wade, 2003; Schradin and Pillay, 2005; Rubenstein and Lovette, 2007; Schradin et al., 2010;

62 Shen et al., 2017), it is no surprise that there is considerable variation in the presence or

63 expression of different types of social behaviors among species.

Comparative studies have been useful in revealing some of the factors favoring the

65 evolution of different types of social behaviors. For example, phylogenetic comparative meta-

66 analyses show that social monogamy is more commonly found in mammalian species that

67 experience low population densities (Lukas and Clutton-Brock, 2013) and genetic monogamy is

68 also more common in mammalian species with low population densities as well as those that

69 exhibit parental care (Lambert et al., 2018). Other comparative studies have shown that

70 cooperative breeding, where subordinate group members care for offspring produced by 
71 dominant breeders, is more likely to be found in mammalian species that live in arid areas

72 (Faulkes et al., 1997; Lukas and Clutton-Brock, 2017) or in avian species that inhabit areas

73 where rainfall is low and unpredictable (Jetz and Rubenstein, 2011). While these studies help

74 illuminate some factors affecting the evolution of social behaviors like social monogamy or

75 cooperative breeding, they typically cannot address the causes of intraspecific variation in

76 sociality.

Sociality is not always expressed to the same level or in the same way within a species

78 and there are often a variety of social strategies within the same species (Lott, 1991; Clutton-

79 Brock, 1989; Getz et al., 1993). The magnitude of variation in the expression of social behavior

80 within a species may not be as large as that among species but it requires explanation

81 nonetheless. Although some studies have investigated some of the proximate causes of

82 individual variation in specific social behaviors such as social monogamy (Young and Wang,

83 2004; Donaldson and Young, 2008; Ophir et al., 2008; Okhovat et al., 2015; Walum and Young,

84 2018) or alloparental care (Dantzer et al., 2017), fewer studies document its fitness

85 consequences. This is not surprising as it requires detailed studies that document the social

86 behavior of individuals and then relate their degree of sociality to their survival and reproduction.

87 Most previous studies on this topic have been conducted in primates where investigators

88 documented how the strength of social relationships ("social bonds") impacted offspring

89 survival, mating success, or longevity (e.g., Silk et al., 2003, 2009, 2010; Schülke, et al., 2010).

90 By investigating the association between social behavior and fitness within a species, we may

91 be able to understand how individuals balance the tradeoffs between the costs and benefits of

92 sociality and therefore obtain an even greater understanding of the evolution of sociality.

93 We characterized the variability of social behavior in individual female and male prairie

94 voles (Microtus ochrogaster) and its association with their mating success, reproductive

95 success, and body condition. Prairie voles are an ideal study system to investigate sociality

96 because both sexes can exhibit natural variation in sociality by displaying different reproductive 
97 strategies (Solomon and Jacquot, 2002). Both females and males can exhibit strategies ranging

98 from pair bonded "residents", with an established territory to non-territorial, unpaired

99 "wanderers" and both are known to switch their strategy over the course of their lifetime (Getz

100 and Hofmann, 1986; Getz et al., 1993; Solomon and Jacquot, 2002; McGuire and Getz, 2010;

101 Shuster et al., 2019). Further, genetic monogamy and social monogamy are distinct with some

102 female-male pairs exhibiting high levels of social and genetic monogamy, some pairs being

103 socially monogamous but not genetically monogamous, and some individuals exhibiting no

104 socially or genetically monogamous behavior or mating patterns (Solomon et al., 2004). Thus,

105 prairie voles may vary in the number and strength of social associations they have with other

106 individuals. For example, a genetically monogamous female and male likely have a very strong

107 social association with each other whereas a wandering individual may have many weak

108 connections with multiple opposite-sex voles. Additionally, voles may vary in the number of

109 social connections they have with other individuals than their partner because under certain

110 environmental conditions, such as high population density (Getz et al., 1993; Cochran and

111 Solomon 2000; Lucia et al. 2008), voles can also form extended family groups when offspring

112 delay dispersal from the natal nest.

113 We characterized the social behavior of individual prairie voles in semi-natural

114 enclosures using an automated monitoring system. Prairie voles were all marked uniquely with

115 passive integrated technology (PIT) tags and their movements were continuously monitored by

116 an array of radio-frequency identification (RFID) antennas. We used patterns of spatial and

117 temporal co-occurrence generated from this system with social network analyses to estimate

118 patterns of social association (unweighted degree). We have previously shown that opposite-

119 sex voles that exhibit close social associations, as generated by this automated method of data

120 collection, are also more likely to be caught in the same trap together, exhibit overlapping home

121 ranges, and show a strong social preference for one another in a choice experiment (Sabol et

122 al., 2018), suggesting that these measures reflect the strength of social associations. Therefore, 
123 we classified individuals with a greater number of social network connections with opposite-sex

124 or same-sex voles as more social, although we note that the valence of these connections

125 (agonistic or affiliative) is not known. We characterized the sociality of all

126 voles throughout the breeding season using social network analyses, recorded their survival

127 through this period and used parentage analyses to quantify their mating success (number of

128 different individuals with which they produced offspring) and reproductive success (number of

129 offspring produced that survived to emergence from the natal nest).

130 We predicted that voles with a greater number of social network connections (i.e., more

131 social) would have higher mating and reproductive success but lower body condition due to the

132 trade-offs associated with high levels of this type of sociality. We only investigated body

133 condition in males because any changes in body mass in females is likely directly related to

134 pregnancy, We predicted that male voles that were more social (had more social network

135 connections) would have lower body condition (mass) because polygyny typically involves

136 energetic costs and risky travel associated with finding female mating partners and interacting

137 with male competitors, which may therefore reduce survival (Blanckenhorn et al 1995; Armitage

138 1998).

140 Methods

141 Study site and study animals

142 All fieldwork was conducted at Miami University's Ecology Research Center in Oxford,

143 Ohio from May to August 2017. Voles were released into two separate 0.1 ha enclosures (33 m

$144 \times 33 \mathrm{~m}$ ). The 20 -gauge sheet metal walls of the enclosures were $75 \mathrm{~cm}$ tall and embedded 45

$145 \mathrm{~cm}$ into the ground to prevent vole movements among enclosures. Enclosure walls were topped

146 with an electrified wire to prevent other small to medium sized mammals (e.g. raccoons and

147 weasels) from entering the enclosures and disturbing traps. Although this system likely

148 prevented many mammalian predators from entering the enclosures, the enclosures were open 
and accessible to avian predators and snakes. We had multiple animals that were confirmed to be depredated by owls and also occasionally saw large snakes within the enclosures. Prior to

151 releasing prairie voles into the enclosures, we live-trapped within the enclosures for 3

152 continuous days to capture any small mammals (e.g. Microtus pennsylvanicus, Peromyscus

153 maniculatus, or Blarina brevicauda) and released them outside of the enclosures.

We released laboratory-bred 7th and 8th generation prairie voles (descended from voles originally captured in Illinois) into two enclosures. The pedigree of the laboratory population was

156 known and to avoid inbreeding, we did not place opposite-sex siblings or parents and their

157 offspring into the same enclosure. All founding voles were sexually mature (> $31 \mathrm{~d}$, Solomon

158 1993) but had never bred. Each enclosure was founded with a different density: Enclosure 1

159 was established by releasing 48 voles (24 females, 24 males) and Enclosure 2 initially

160 contained 24 voles (12 females, 12 males). These represented densities of 480 voles/ha and

161240 voles/ha respectively, which were within the range of vole densities observed in natural

162 populations (10 to 600 voles/ha: Getz et al., 1994; Getz et al., 2001). The different starting

163 density was employed to assess the role of density on vole social behavior but, as shown below

164 (Fig. 1), vole density in Enclosure 1 (high density) decreased over the course of the field

season. Additionally, we do not find or report an effect of density on vole social behavior in this dataset, only whether there is a difference between the enclosures in general for the whole season. The vegetation within enclosures consisted primarily of perennial grasses and forbs,

168 which provided food and cover. Voles were not provided with supplemental food besides the 169 cracked corn, a low-quality food, used to bait the live-traps.

\section{Recording social behavior of voles}

171 Prior to release into the enclosures, all founding voles were implanted with a PIT tag

172 (Biomark: Boise, Idaho) with a unique identification number. Once released, we monitored our

173 populations through a proprietary system of 12 RFID antennas connected to a central recording

174 station (BioMark RM310/SM303, Boise, ID) that we have used previously to create social 
175 networks of prairie voles and infer their social associations (Sabol et al., 2018). These antennas

176 were placed within the enclosures in two different $3 \times 4$ arrays (Figure A1) that were rotated

177 from array 1 to array 2 every 3 days and moved from one enclosure to the other every 6 days

178 during the 14-week study. The antennas recorded the individual PIT tag numbers of each vole

179 that passed within $15 \mathrm{~cm}$ of the antenna once a second for the entire time the animal was within

180 this radius of the antenna. When multiple tags were within the $15 \mathrm{~cm}$ radius, the system

181 alternated in recording the tag numbers so that both could be detected. This allowed us to

182 record the natural movements and social associations of individuals in both populations, which

183 we have previously shown to be comparable and more detailed than traditional methods of

184 recording social associations in these populations (Sabol et al., 2018).

185 Monitoring vole reproduction

We live-trapped both enclosures by placing two Ugglan live- traps (Granhab, Hillerstorp,

187 Sweden), baited with cracked corn, per grid stake on a 5 x $5 \mathrm{~m}$ trapping grid. These live-traps

188 had a metal cover over the top to shield voles from the elements while in the traps. At the

189 beginning of the experiment, we live-trapped nearly every day, setting traps in the evening

190 (2230-2300 h) and checking them approximately two hours later and then leaving them set to be

191 checked the next morning $(0700 \mathrm{~h})$. After the first two weeks of the experiment, we set traps

192 approximately 3 times per week per enclosure (Monday, Wednesday, and Friday) alternating

193 between the two enclosures so that voles in each enclosure were trapped three days over the

194 course of two weeks. Traps were set in the evening (2230-2300 h) and checked the following

195 morning at $0700 \mathrm{~h}$. If we needed to put radiocollars on voles (see below), we also set traps from

$1961800-1900 \mathrm{~h}$ and then checked them the same evening from 2000-2100 h. Prairie vole gestation

197 and time to weaning are each approximately 21 days (Richmond \& Conaway, 1969) and,

198 therefore, the offspring produced within the enclosures usually first emerge from the nest

199 approximately 5-6 weeks after the adults are released (N. Solomon, B. Keane, personal

200 observations). We therefore stopped setting traps overnight from week 6-14 of the experiment 
201 to ensure that offspring would not spend an entire night without their parents. During this time,

202 we set traps in the evening (2230-2300 h) and checked them approximately two hours later but

203 did not leave traps set overnight. Finally, we did not trap when there were severe thunderstorms

204 and made up this trapping session on a different day during the week, if possible. We

205 supplemented this regular grid trapping by placing additional traps at nest entrances after we

206 located them using VHF telemetry and/or UV powder tracking (Lemen and Freemen, 1985).

207 During each capture, we identified each individual using a handheld PIT tag reader,

208 recorded the location where the vole was live-trapped, the other individuals caught in the same

209 trap, body mass (g, with Pesola spring scale), and assessed reproductive condition. Males were

210 recorded as being either scrotal or non-scrotal depending on the development of the testes.

211 Females were recorded as non-reproductive, pregnant, or lactating depending on whether

212 developing fetuses could be felt or if nipples were pronounced. Once offspring were live trapped

213 for the first time, we gave them a unique toe clip until they were large enough (>25 $\mathrm{g}$ ) to be PIT

214 tagged. Reproductive success of adult voles was estimated as the total number of offspring they

215 produced that we were able to trap.

216 Parentage analyses

217 Before adult voles were released into the enclosures, we collected a small piece of ear

218 tissue and stored it in $70 \%$ ethanol in a $-80^{\circ} \mathrm{C}$ freezer. When offspring were trapped for the first

219 time, the tissue from the identifying toe clip was saved and temporarily stored in a $-20^{\circ} \mathrm{C}$ freezer

220 until samples could be moved to a $-80^{\circ} \mathrm{F}$ freezer. We extracted DNA using DNeasy Blood and

221 Tissue kits (Qiagen). We followed the manufacturer's protocol except that tissue samples were

222 incubated at $70^{\circ} \mathrm{C}$, DNA was eluted in $100 \mu \mathrm{l}$ of molecular grade water instead of $200 \mu \mathrm{l}$ of buffer

$223 \mathrm{AE}$, and DNA samples were incubated at room temperature for 5 minutes instead of 1 minute.

224 Once DNA was extracted, we genotyped the samples for all adults and offspring at seven

225 microsatellite loci (Keane et al., 2007). We then ran an allele frequency analysis on the population

226 of voles in each enclosure separately using Cervus 3.0.7 (see Mabry et al., 2011 for full details). 
227 To determine parentage, we used Cervus 3.0.7 parentage analyses with known sexes, which

228 calculates a likelihood ratio for each potential mother and father in order to determine the most

229 likely biological parents in the population for each offspring (Mabry et al., 2011). We were able to

230 determine both parents (trio confidence level) with a 95\% confidence level for $33 / 41$ (80.5\%)

231 offspring, so only these 33 offspring were included in the analyses of mating and reproductive

232 success.

233 Ethical Note

$234 \quad$ All procedures involving live animals were in accordance with the guidelines provided by

235 the American Society of Mammalogists for the use of wild mammals in research (Sikes et al.,

236 2016) and were approved by Miami University's Institutional Animal Care and Use Committee

237 (protocol number 979) as this was where all work with live animals occurred.

238 Statistical Analyses

All statistical analyses were done in $\mathrm{R}$ version 3.4.1 (R Core Team, 2017). Figure 1 was

240 made in $\mathrm{R}$ while all other figures were made in ggplot2 version 2.2.1 (Wickham, 2009). All linear

241 models and generalized linear models were run in R. For all models listed below, we assessed

242 collinearity among the predictor variables using variance inflation factors (VIFS) in the package

243 car, version 3.0-0 (Fox and Weisberg, 2011).

244 Density and Body Mass

245 Population density was calculated based on the number of unique individuals caught

246 within each two-week period (over each two-week period both enclosures were trapped with

247 equal effort except for occasional cancellations due to weather). To investigate population

248 density over the course of the field season, we used a linear model with density (log-

249 transformed with base 10 to improve normality of residuals) with the fixed effects of enclosure

250 and weeks in the study and the interaction of these terms. Sex ratio was calculated by dividing

251 the number of adult males by the number of total adults for each two-week period. We used a

252 binomial generalized linear model to investigate sex ratio with the fixed effects of enclosure and 
253 weeks in the study and the interaction of these terms. VIFs for all non-interaction terms were all

$254<3.57$

255 Social Network Analyses

256 We measured the number of social connections (unweighted degree, hereafter degree)

257 between same-sex or opposite-sex voles based on co-occurrence data from the RFID

258 antennas. Individuals with a high degree would have had instances of spatial and temporal co-

259 occurrence with many other voles whereas those with a low degree had few. We conducted all

260 social network analyses using the R package asnipe version 1.1.4 (Farine, 2017b). In order to

261 generate our social networks, we took the PIT tag readings from the RFID antennas and ran

262 them through a Gaussian Mixture Model with each day labeled separately (Psorakis et al.,

263 2012). This model goes through the raw data of the PIT tag readings and creates groups based

264 on when tag readings at the same antenna are clumped throughout time. Therefore, there is not

265 a uniform time period used to create these groups, they are based on how our data were

266 distributed over time. This model uses clusters of tag readings as "centres of mass" where data

267 are concentrated and then determines the groups based on the amount and distribution in time

268 of tag readings in each cluster to determine where to split groups (Psorakis et al., 2012). The

269 duration of these group events ranged from 0 seconds (so voles were both at the antenna at the

270 same time) to 66,161 seconds with an average of $655.2 \pm 3,352.8$ seconds. This then creates a

271 group by individual matrix where being in the same spatial and temporal "group" counts as an

272 association between individuals. As we were only interested in the number of connections each

273 individual had (not the strength of these connections), we used a binary, unweighted

274 measurement of degree where any non-zero association was counted as a "1". Thus, anytime

275 we refer to the number of social connections in this paper, we calculated this using the

276 unweighted degree. For more details about the construction of the social networks see Sabol et

277 al. (2018).

278 Reproductive Success Models 
For all models including the number of mates (mating success) or the number of offspring produced that survived to emergence from the natal nest (reproductive success) as the

281 response variable, we used Poisson generalized linear models. For each response variable we

282 ran two models using social network data, one including all social connections in order to

283 investigate sociality overall and one including only the opposite-sex social connections. These

284 models all had fixed effects of the number of social connections, the interaction of this with sex,

285 the number of social connections squared to assess non-linear effects of social connections on

286 mating success or reproductive success (one model including all connections and another for

287 each response variable including only opposite-sex connections), the interaction of this with sex,

288 enclosure, and survival (calculated as the proportion of the field season the individual survived

289 based on last detection). To test if mating success and reproductive success were related, we

290 ran a separate model with the number of offspring produced as the response variable and fixed

291 effects of the number of mates with which individuals produced offspring, the interaction of this

292 with sex, and enclosure. None of the GLMs were over-dispersed as all the dispersion

293 parameters were <1, which we tested using R package AER version 1.2.5 (Kleiber and Zeil,

294 2008). VIFs were all < 3.5 except interaction and squared terms, which were predictably high.

295 Body Mass

To investigate body mass, we calculated the average body mass for each male vole for

297 the entire field season (range 1-19 measurements, average 7.75 measurements). Females

298 were not included because we were using body mass as a proxy for body quality, and female

299 mass would be affected by both pregnancy status and body condition. We then used a general

300 linear model for average body mass with the number of social connections (one model including

301 all connections another including only opposite-sex connections), the number of social

302 connections squared (one model including all connections another including only opposite-sex

303 connections), enclosure, survival (calculated as the proportion of the field season the individual

304 survived based on last detection), and the number of mass measurements we had for each 
305 individual. We visually assessed the distribution of the data and residuals for normality. VIFs were all $<2.2$ except interaction or squared terms and survival. However, when survival was excluded from the model, VIFs for all of the other terms were $<3.5$ except interactions and squared terms. Including survival did not alter the statistical significance of any of the results shown below so we left it in.

Randomized Models

For every model that included unweighted degree (the number of social connections),

312 we used the network permutation method in asnipe (Farine, 2017b). This method is useful

313 because it helps control for the fact that social network data are not independent. This method

314 also allows us to investigate our hypotheses more specifically by allowing us to test if the

315 observed relationships are significantly different from random networks with the same structure

316 as our social networks (see Farine et al., 2015; Spiegel et al. 2017 for other similar uses of this

317 method). The network permutation method takes a piece of data from the group by individual

318 matrix and swaps it for a different individual (Farine, 2013). Specifically, we ran 10,000

319 randomized models where each time another piece of data from the individual by group matrix

320 was swapped. We also restricted swaps to only voles in the same enclosure that were recorded

321 on the RFID antennas during the same day to control for voles that did not survive the entire

322 season. Further, for the opposite-sex networks we restricted swaps to include only voles of the

323 same sex so that we were only comparing our opposite-sex network to other opposite-sex

324 networks, not all possible combinations. We then compared the regression coefficients from the

325 model for each variable that includes a social network statistic to corresponding b-values from

326 randomized networks and calculated a new $P$-value based on the number of randomized

327 models that produced a b-value with a higher absolute value than the absolute value of the

328 observed model. Therefore, our $P$-value shows us whether the relationship we have observed is 329 stronger than the relationship from 10,000 randomizations of our dataset (Farine, 2013). We ran 330 each set of randomizations three times to ensure that the $P$-values were consistently significant 
331 in each of the randomizations. We present all three $P$-values from these randomizations and,

332 conservatively, only consider that a relationship is statistically significant if all three

333 randomizations revealed $P$-values $<0.05$.

$335 \quad$ Results

336 Enclosure density \& adult sex ratio

The number of adult voles in each enclosure declined over the course of the field season due to mortality (effect of weeks in the study, $b=-0.14, \mathrm{SE}=0.012, \mathrm{t}_{12}=-11.45, P<$ 0.0001, Fig. 1a, note estimates on $\log _{10}$ scale) and the significant interaction between weeks in

340 the study and enclosure indicated that vole density decreased more strongly in the high density 341 enclosure than in the low density enclosure (enclosure $\mathrm{x}$ weeks: $\mathrm{b}=0.083, \mathrm{SE}=0.017, \mathrm{t}_{12}=$ $3424.79, P=0.00045)$. For example, the starting density of voles in the high-density enclosure was 343480 voles/ha (week 0 in Fig. 1a) but was reduced to 200 voles/ha in the middle of the 344 experiment (week 8 in Fig. 1a) and to 60 voles/ha at the end of the experiment (week 14 in Fig. 345 1a). The starting density in the low-density enclosure was 240 voles/ha (week 0 in Fig. 1a) but 346 was reduced to 180 voles/ha in the middle of the experiment (week 8 in Fig. 1a) and 100 347 voles/ha at the end of the experiment (week 14 in Fig. 1a). In total, only $12.5 \%$ of voles in the 348 high-density enclosure were still alive at the end of the experiment whereas $41.7 \%$ of the voles 349 in the low-density enclosure were still alive at the end of the experiment.

350 Unlike density, adult sex ratio did not differ significantly during the course of the 351 experiment (effect of time, $b=-0.04, \mathrm{SE}=0.16, \mathrm{z}=-0.26$, df $=12, P=0.80$, Fig. $1 \mathrm{~b}$ ) or between

352 the two enclosures (effect of enclosure, $b=0.15, \mathrm{SE}=1.84, \mathrm{z}=0.082$, df $=12, P=0.94$, Fig. 353 1b). 
Overall, both female and male voles that had an intermediate (i.e., the middle of the range of observed values) number of social connections produced offspring with a greater number of different mates (i.e., had higher mating success). In the model considering all social interactions with same- and opposite-sex individuals, voles that had an intermediate number of social connections (degree) with all possible individuals had higher mating success (effect of social connections ${ }^{2}: \mathrm{b}=-0.012, \mathrm{z}=-0.88, P$-values from randomized networks $=0.012,0.0093$, $<0.0001$, Table 1, Fig. 2a) but this relationship was slightly different between the sexes (sex $x$ social connections ${ }^{2}: \mathrm{b}=0.014, \mathrm{z}=0.94, P=0.010,0.0018,<0.0001$, Table 1 , Fig. $2 \mathrm{a}$ ). In both females and males, those with an intermediate number of social connections had the highest mating success, therefore the interaction with sex and the number of social connections ${ }^{2}$ on mating success seemed to be largely due to males having slightly more overall social connections than females while female mating success peaked at a lower number of social connections (Fig. 2a). There is also a qualitative difference in the shape of the curve, with female mating success peaking at a lower number of social connections but then dropping off more steeply, while male mating success peaked at a higher number of social connections but declined more gradually (Fig. 2a).

The same relationship was true when only opposite-sex connections were considered.

372 Mating success was highest for female and male voles with an intermediate number of

373 opposite-sex social connections (social connections ${ }^{2}$ : $b=-0.028, z=-1.01, P=0.0011,0.0035$,

3740.001 , Fig. $2 b$ ), although the magnitude of this effect slightly differed between the sexes (sex $x$ 375 social connections ${ }^{2}: b=0.057, z=1.36, P=<0.0001,<0.0001,0.0068$, Table 1 , Fig. $\left.2 b\right)$. This

376 latter difference between the sexes seems to be driven by the difference in the number of social 377 connections between the sexes with females tending to have slightly more social connections 378 than males. success than individuals in the higher density enclosure (from model for all social connections: b 
$=1.89, z=4.55, P<0.0001$; from model for all opposite-sex social connections: $b=2.03, z=$

4.72, $P<0.0001$, Table 1). Individuals that survived in the enclosures for longer had higher mating success (all social connections: $\mathrm{b}=2.07, \mathrm{z}=2.33, P=0.020$; opposite-sex social connections: $\mathrm{b}=1.75, \mathrm{z}=2.09, P=0.037$, Table 1$)$. voles produced more offspring that survived to emergence from the natal nest $(b=-0.0085, z=$ $-0.77, P=0.002,0.035,0.015$, Table 2 , Fig. 3a). This result did not consistently differ by sex (sex x social connections ${ }^{2}: b=0.0082, z=0.63, P=0.021,0.11,0.022$, Table 2, Fig. $3 a$ ). However, when only considering opposite-sex social connections (Fig. 3b), these relationships were not significant. The number of offspring that voles produced was not related to the number of opposite-sex social connections for males or females (effect of opposite-sex social connections: $b=0.15, z=0.52, P=0.20,0.53,0.54$, Table 2, Fig. $3 b)$ and voles with an intermediate number of opposite-sex social connections did not produce significantly more offspring (effect of opposite-sex social connections ${ }^{2}: b=-0.022, z=-0.93, P=0.14,0.099,0.12$, Table 2, Fig. 3b). This relationship did not consistently vary with sex, as the difference between

397 the sexes for opposite-sex connections ${ }^{2}$ where the inverted u-shaped relationship was slightly

398 lessened in males and was not significant in all three sets of randomizations (sex x opposite-sex 399 connections $^{2}: \mathrm{b}=0.038$, z-value $=0.92, P=0.063,0.010,0.012$, Table 2 , Fig $\left.3 b\right)$ and the

400 difference between opposite sex connections between males and females where males tended

401 to have fewer connections than females was also not significant in all three sets of

402 randomizations (sex $x$ number of opposite-sex connections: $b=-0.39, z=-1.04, P=0.047$,

$4030.024,0.11$ ). Male and female voles that survived for longer produced more offspring (all social

404 connections: $b=2.49, z=3.03, P=0.0025$; opposite-sex social connections: $b=2.19, z=2.83$, $405 P=0.0046$, Table 2). 

number of individuals) in both females and males. Individuals with more mates produced more

409 offspring that survived to emergence from the natal nest $(b=0.64, z=4.88, P<0.0001$, Table 3,

410 Fig. 4) and this relationship did not differ by sex as the interaction between number of mates

411 and sex was not significant $(b=-0.093, z=-0.56, P=0.58$, Table 3$)$. Individuals in the lower

412 density enclosure had higher reproductive success than individuals in the higher density

413 enclosure $(b=1.53, z=3.37, P=0.00076$, Table 3$)$.

\section{Effects of sociality on body mass}

416 (effect of social connections ${ }^{2}$ : $\mathrm{b}=-0.066, \mathrm{t}=-2.36, P=<0.0001,<0.0001,<0.0001$, Table 4,

417 Fig. 5a). This relationship was also significant when only opposite-sex connections were

418 considered (effect of opposite-sex social connections ${ }^{2}$ : $b=-0.20, t=1.54, P=0.0024,0.0031$,

4190.0005 , Table 4, Fig. 5b). Average body mass was not significantly different between the two

420 enclosures (all social connections: $\mathrm{b}=-1.03, \mathrm{t}=-0.40, P=0.69$; opposite-sex social

421 connections: $\mathrm{b}=-0.16, \mathrm{t}=-0.058, P=0.95$, Table 4). Survival did not predict average body

422 mass in either model (all social connections: $b=13.37, t=1.52, P=0.14$; opposite-sex social

423 connections: $\mathrm{b}=10.80, \mathrm{t}=1.22, P=0.23$, Table 4). Although the number of measurements we

424 obtained to calculate each individual's average body mass varied (Fig. 5), it did not affect our

425 measure of average body mass in either model (all social connections: $\mathrm{b}=-0.12, \mathrm{t}=-0.20, P=$

426 0.84; opposite-sex social connections: $\mathrm{b}=-0.20, \mathrm{t}=-0.31, P=0.76$, Table 4).

\section{Discussion}

As expected, both female and male prairie voles varied in the frequency of social

430 interactions, as reflected in our social network analyses that quantified social network degree 
431 using the frequency of temporal and spatial co-occurrence generated by our RFID system.

432 Female and male voles with an intermediate number of social interactions had the greatest

433 mating success and produced the greatest number of offspring, though the latter was only the

434 case when we included all interactions with other voles, and not when only opposite-sex

435 connections were considered.

436 We tested our predictions about the fitness benefits of sociality using two different sets

437 of social network data, one including social connections with all voles and one only including

438 connections with opposite-sex individuals. This allowed us to investigate the potential costs and

439 benefits of sociality overall (e.g., social interactions of a female vole with other females and

440 males) as well as specifically social interactions between opposite-sex individuals, which may

441 be more directly relevant to mating and reproductive success. We found that voles with an

442 intermediate number of social connections with all voles (i.e., more social) had significantly

443 higher mating and reproductive success whereas voles with an intermediate number of

444 opposite-sex connections had significantly higher mating success but not higher reproductive

445 success. The process of running the randomizations was somewhat different for the two sets of

446 networks (all social connections or just opposite-sex connections), which could contribute to the

447 observed differences in the results between the two sets of networks. We limited permutations

448 for the opposite-sex network to within the same sex (where female social network data were

449 being swapped for another female and male data were being swapped for another male) so

450 these swaps could only be done between approximately half as many individuals each time. By

451 keeping the sex of each individual in the association consistent, the structure of the

452 randomizations was more like the data it was being compared to instead of comparing all

453 possible connections (Farine, 2017a). However, since the permutations were done on the raw

454 data and then we pulled only the opposite-sex connections from these networks, it is possible

455 that some of the 10,000 permutations affected the same-sex connections in the raw data

456 (therefore changing a social interaction that is not included in data used for the linear model) 
457 and therefore may not have changed the estimate for the relationship between opposite-sex

458 connections and the response variable every time, whereas in the models with all connections

459 included, a relevant social interaction would have changed every time, which would then change

460 the model estimate for the relationship between social connections and the response variable

461 some amount every time. Since we ran such a large number of these permutations, this may not

462 have affected the overall result of the tests, but it is a limitation of the method.

463 Using our measure of sociality, our results suggest the possibility of stabilizing selection

464 on sociality because voles that co-occurred spatially and temporally with very few or very many

465 conspecifics had the lowest mating success and, at least when considering all social

466 connections, had the lowest reproductive success. Both McGuire et al. (2002) and Solomon and

467 Keane (2018) showed that large social groups do not increase female reproductive success.

468 McGuire et al. (2002) also showed that female prairie voles that lived in large groups had fewer

469 offspring survive to 12 or 30 days of age. Similarly, Solomon and Keane (2018) showed that

470 females did not benefit from living in large social groups in two other natural populations. These

471 studies are consistent with our results that a very large number of social connections (which

472 should occur in large social groups) does not increase reproductive success of breeding

473 females, and our results show that this is also true for males.

474 We only recorded the association between one measure of sociality and mating and

475 reproductive success in one year and the effects of the number of social connections on fitness

476 could be altered when environmental conditions change. For example, the Female Dispersion

477 Hypothesis would predict that if our measure of sociality reflects the socially monogamous

478 behavior of male prairie voles, our measure of sociality should be positively correlated with male

479 mating success when females are spatially clumped as males that have more social

480 connections with clumped females should have higher mating success (Shuster and Wade,

481 2003; Dobson et al., 2010; Lukas and Clutton-Brock, 2013). In natural populations of prairie

482 voles, density is quite variable across years (Getz et al., 1993, 2001) and some previous 
483 observational studies of prairie voles in field settings suggested that socially monogamous

484 behavior is more common at low densities (McGuire et al.,1990; Solomon et al., 2009; but see

485 Getz and McGuire,1993). There is also some evidence that resource distribution may impact the

486 mating strategy of prairie voles and this effect may be mediated through its influence on density

487 (Streatfeild et al., 2011). This suggests the possibility that selection on the social behavior of

488 prairie voles varies among years due to changes in population or female density but additional

489 multi-year studies measuring a broader array of social behaviors in free-living voles are needed

490 to test this prediction.

491 One possible explanation for an intermediate level of sociality being associated with the

492 highest mating success and potentially highest reproductive success is that this reflects a

493 tradeoff between devoting time to social interactions (although we do not know the type of social

494 interaction occurring) with other voles and time to other behaviors like foraging and parental

495 care. Although high levels of sociality can have beneficial effects on individual fitness, it may

496 also carry costs for an individual's health or physical condition (Nunn et al., 2015). Indeed, we

497 found that male voles with the most social connections had the lowest body mass, suggesting

498 that there may be a reduction in body condition associated with a very high level of sociality.

499 This could reflect the energetic costs associated with having many social connections or living in

500 a large group (e.g., Lutermann et al., 2013), or these could be agonistic interactions with males

501 on neighboring territories, resulting in males investing more time in territory defense than males

502 with fewer neighbors. Why males with very few social connections were also lighter in body

503 mass is not clear but these males may have been of lower phenotypic quality given that they

504 had few social connections, low body mass, and low mating and reproductive success.

505 Alternatively, having fewer social connections could result in a loss of body mass if these males

506 had no assistance in territory defense and thus, expended more energy than males with more

507 social connections (e.g., having a female social partner). Females likely face many of these 
same tradeoffs, but as we did not test quality in females (due to changes in mass being linked to pregnancy) and so further study is needed to investigate this relationship in females.

511 too many opposite-sex conspecifics. One possible explanation is that individuals with an

512 intermediate number of social connections may better balance the trade-off between the

513 number and quality or strength of social relationships. For example, individuals with the highest

514 social network degree may just have many weak social or agonistic connections, which may not

515 result in more matings or increased reproductive success. Individuals with an intermediate

516 social network degree may have more affiliative social connections that are strong enough to

517 result in matings than voles at either extreme. This also is reflected in the fact that voles have

518 many more social connections than actual mating partners (Fig. 2), where the range of the

519 number of mates varies from 0 to 4 while the range of the number of social connections with

520 opposite-sex individuals is from 0 to 14 . This is supported by studies of the association between

521 the strength of social connections and fitness in cercopithecine primates (baboons) where

522 females with strong social bonds with other females in their group have higher offspring survival

523 (Silk et al., 2003, 2009) or longevity (Silk et al., 2010). Similar relationships between the

524 strength of social bonds and fitness have also been found in male primates; male Assamese

525 macaques (Macaca assamensis) with strong social bonds to other males (including unrelated

526 males) sired more offspring than those with fewer strong bonds with other males (Schülke, et

527 al., 2010). As the number of social connections increase, the strength of association of each of

528 these social connections may decline (Whitehead 2008), thus prairie voles may be constrained

529 by the number of social connections in which they can invest enough time to result in successful

530 mating or rearing of offspring given that prairie voles exhibit biparental care. Individuals that can

531 best balance this trade-off between the number and strength of social connections may have

532 the highest mating and reproductive success. 
It is of course likely that the fitness benefits of the quantity versus quality of social

534 connections may vary according to whether the modal social structure of the species is group-

535 living (such as primate species mentioned above where strong social bonds increase fitness) or

536 its mating system. For example, Ryder et al. (2009) found a positive association between the

537 number of social connections (social network degree) and number of offspring sired in male

538 manakins. As this is a lekking species, coordinated male displays may make male-male

539 connections a more important factor for mating success than in prairie voles. Additionally, the

540 short-term coalitions at leks may make the strength of the relationship less important than in

541 species like prairie voles. Studies like these are rare and so future studies across a broader

542 array of species with different mating systems will be needed to fully characterize the

543 relationship between the number and strength of social connections and measures of fitness.

544 Doing so will help provide insight into how individuals within a species balance the fitness costs

545 and benefits of social behavior, thereby providing a complementary approach to comparative

546 studies regarding the evolution of social behavior.

548 Acknowledgements

549 We thank the Ecology Research Center (ERC) at Miami University for permitting us to conduct

550 this study and Jeremy Fruth, field manager at the ERC, for coordinating the project. We also

551 thank James Lichter, Sage Sparks, Caleb Collins, Tony Leon, Meghan Charles, and Staci

552 Burrow for assisting on the project. We also thank the American Society of Mammalogists

553 (Grant-in-Aid of Research to A. Sabol) and University of Michigan (B. Dantzer) for funding.

555 Data Statement

556 All raw data and R code are available under CC-BY from FigShare at the link

557 https://figshare.com/projects/How does individual variation in sociality influence fitness in $p$

558 rairie voles /71969). 


\section{Literature Cited}

560 Alexander, R. D. (1974). The evolution of social behaviour. Annual Review of Ecology, Evolution,

561 and Systematics, 5, 325-383.

562 Armitage, K. B. (1998). Reproductive strategies of yellow-bellied marmots: Energy conservation

563 and differences between the sexes. Journal of Mammalogy, 79, 385-393.

564 Blanckenhorn, W. U., Preziosi, R. F. \& Fairbairn, D. J.(1995). Time and energy constraints and

565 the evolution of sexual size dimorphism - to eat or to mate? Evolutionary Ecology, 9, 369-381.

566 doi:10.1007/BF01237760

567 Clutton-Brock, T. H. (1989). Review lecture: mammalian mating systems. Proceedings of the

568 Royal Society B, 236, 339-372.

569 Clutton-Brock, T.H., Brotherton, P.N.M., Smith, R., Mclllrath, G.M., Kansky, R., Gaynor, D., O'rian,

570 M.J., Skinner, J.D. (1998). Infanticide and expulsion of females in a cooperative mammal.

571 Proceedings of the Royal Society B, 265, 2291-2295.

572 Cochran, G. R., \& Solomon, N. G. (2000). Effects of food supplementation on the social

573 organization of prairie voles (Microtus ochrogaster). Journal of Mammalogy, 81, 746-757.

574 Côté, I.M., \& Poulinb R. (1995). Parasitism and group size in social animals: a meta-analysis.

575 Behavioral Ecology, 6, 159-165.

576 Creel, S., Dantzer, B., Goymann, W. \& Rubenstein, D.R. (2013). The ecology of stress: effects of

577 the social environment. Functional Ecology, 27, 66-80.

578 Dantzer, B., Goncalves, I.B., Spence-Jones, H.C., Bennett, N.C., Heistermann, M., Ganswindt, A.,

579 Dubuc, C., Gaynor, D., Manser, M.B., Clutton-Brock, T.H. (2017). The influence of stress

580 hormones and aggression on cooperative behaviour in subordinate meerkats. Proceedings of

581 the Royal Society B, 284, 20171248.

582 Dobson, F. S., Way, B. M., \& Baudoin C. (2010). Spatial dynamics and the evolution of social

583 monogamy in mammals. Behavioral Ecology, 21, 747-752. doi:10.1093/beheco/arq048 
584 Donaldson, Z. R., \& Young, L. J. (2008). Oxytocin, vasopressin, and the neurogenetics of

585 sociality. Science, 322, 900-904. doi:10.1126/science.1158668

586 Eisenberg, J.F., Muckenhirn, N.A., Rudran, R. (1972). The relation between ecology and social 587 structure in primates. Science, 176, 863-874.

588 Emlen, S.T. (1984). Cooperative breeding in birds and mammals. Behavioural Ecology: An

589 Evolutionary Approach. J.R. Krebs and N.B. Davies (Eds.), pp. 305-339, Blackwell, Oxford.

590 Emlen, S.T. (1994). Benefits, constraints and the evolution of the family. Trends in Ecology and

591 Evolution, 9, 282-285.

592 Ewald, P. W. (1994) Evolution of Infectious Disease. Oxford: Oxford University Press.

593 Farine, D. R. (2013). Animal social network inference and permutations for ecologists in R using

594 asnipe. Methods in Ecology and Evolution, 4, 1187-1194. doi:10.1111/2041-210X.12121

595 Farine, D. R., Firth, J. A., Aplin, L. M., Crates, R. A., Culina, A., Garroway, C.J., Hinde, C. A.,

596 Kidd, L. R., Milligan, N. D., Psorakis, I., Radersma, R., Verhelst, B., Voelkl, B., \& Sheldon, B. C.

597 (2015). The role of social and ecological processes in structuring animal populations: a case

598 study from automated tracking of wild birds. Royal Society Open Science, 2.

599 doi:10.1098/rsos.150057

600 Farine, D. R. (2017a). A guide to null models for animal social network analysis. Methods in

601 Ecology and Evolution, 8, 1309-1320. doi:10.1111/2041-210X.12772

602 Farine, D. R. (2017b). asnipe: Animal Social Network Inference and Permutations for Ecologists.

603 R package version 1.1.4. Available online at: https://CRAN.R-project.org/package=asnipe

604 Faulkes, C. G., Bennett, N. C., Bruford, M. W., O'brien H. P., Aguilar G. H., \& Jarvis, J. U. M.

605 (1997). Ecological constraints drive social evolution in the African mole-rats. Proceedings of the

606 Royal Society of London B: Biological Sciences, 267. doi:10.1098/rspb.1997.0226

607 Fox, J., \& Weisberg, S. (2011). An \{R\} Companion to Applied Regression, Second Edition.

608 Thousand Oaks CA: Sage. URL: http://socserv.socsci.mcmaster.ca/jfox/Books/Companion 
609 Getz, L. L., \& Hofmann, J. E. (1986). Social organization in free-living prairie voles, Microtus

610 ochrogaster. Behavioral Ecology and Sociobiology, 18, 275-282.

611 Getz, L. L., Hofmann, J. E., McGuire, B., \& Dolan, T. W. III. (2001). Twenty-five years of

612 population fluctuations of Microtus ochrogaster and M. pennsylvanicus in three habitats in east-

613 central Illinois. Journal of Mammalogy, 82, 22-34.

614 Getz, L. L., \& McGuire, B. (1993). A comparison of living singly and in male-female pairs in the

615 prairie vole, Microtus ochrogaster. Ethology, 94, 265-278. doi:10.1111/j.1439-

$616 \underline{0310.1993 . t b 00444 . x}$

617 Getz, L. L., McGuire, B., Pizzuto, T., Hofmann, J. E., \& Frase, B. (1993). Social organization of

618 the prairie vole (Microtus ochrogaster). Journal of Mammalogy. 74, 44-58. doi:

$619 \quad 10.2307 / 1381904$

620 Getz, L. L., McGuire, B., Hofmann, J., Pizzuto, T., \& Frase, B. (1994). Natal dispersal and

621 philopatry in prairie voles (Microtus ochrogaster): settlement, survival, and potential reproductive

622 success. Ethology Ecology \& Evolution, 6, 267-284.

623 Hatchwell, B. J., \& Komdeur, J. (2000). Ecological constraints, life history traits and the evolution

624 of cooperative breeding. Animal Behaviour, 59, 1079-1086. doi:10.1006/anbe.2000.1394

625 Jetz, W., \& Rubenstein, D. R. (2011). Environmental uncertainty and the global biogeography of

626 cooperative breeding in birds. Current Biology, 21, 72-78. doi:10.1016/j.cub.2010.11.075.

627 Kappeler, P. M., Cremer, S., \& Nunn, C. L. (2015). Sociality and health: impacts of sociality on

628 disease susceptibility and transmission in animal and human societies. Philosophical

629 Transactions of the Royal Society B: Biological Sciences, 370. doi:10.1098/rstb.2014.0116

630 Keane, B., Bryant, L., Goyal, U., Williams, S., Kortering, S. L., Lucia, K. E., Richmond, A. R., \&

631 Solomon, N. G. (2007). No effect of body condition at weaning on survival and reproduction in

632 prairie voles. Canadian Journal of Zoology, 85, 718-727. 
633 Kleiber, C., \& Zeileis, A. (2008). Applied econometrics with R. New York: Springer-Verlag. ISBN 978-0-387-77316-2. https://CRAN.R-project.org/package=AER" https://CRAN.R-

635 project.org/package=AER

636 Krause, J., \& Ruxton, G. D. (2002). Living in groups. Oxford: Oxford University Press.

637 Lambert, C. T., Sabol, A. C., \& Solomon, N. G. (2018). Genetic monogamy in socially

638 monogamous mammals is primarily predicted by multiple life history factors: a meta-analysis.

639 Frontiers in Ecology and Evolution, 6, 139. doi:10.3389/fevo.2018.00139

640 Langwig, K. E., Frick, W. F., Bried, J. T., Hicks, A. C., Kunz, T. H. \& Marm Kilpatrick, A. (2012),

641 Sociality, density-dependence and microclimates determine the persistence of populations

642 suffering from a novel fungal disease, white-nose syndrome. Ecology Letters, 15: 1050-1057.

643 doi:10.1111/j.1461-0248.2012.01829.x

644 Lemen, C. A., \& Freeman, P. W. (1985). Tracking mammals with fluorescent pigments: a new

645 technique. Journal of Mammalogy, 66, 134-136.

646 Lott, D. F. (1991). Intraspecific variation in the social systems of wild vertebrates. Cambridge:

647 Cambridge University Press.

648 Lucia, K. E., Keane, B., Hayes, L. D., Lin, Y. K., Schaefer, R. L., \& Solomon, N. G. (2008).

649 Philopatry in prairie voles: an evaluation of the habitat saturation hypothesis. Behavioral.

650 Ecology, 19, 774-783. doi: 10.1093/beheco/arn028

651 Lukas, D., \& Clutton-Brock, T. H. (2013). The evolution of social monogamy in mammals.

652 Science, 341, 526 -530. doi:10.1126/science.1238677

653 Lukas, D., \& Clutton-Brock, T. H. (2017). Climate and the distribution of cooperative breeding in

654 mammals. Royal Society Open Science, 4. doi:10.1098/rsos.160897

655 Lukas, D., \& Huchard, E. (2014). The evolution of infanticide by males in mammalian societies.

656 Science, 346, 841-844. doi:0.1126/science.1257226 
657 Lutermann, H., Bennett, N. C., Speakman, J. R., \& Scantlebury, M. (2013). Energetic benefits of

658 sociality offset the costs of parasitism in a cooperative mammal. PLOS ONE, 8, e57969.

659 doi:10.1371/journal.pone.0057969

660 Mabry, K. E., Streatfeild, C. A., Keane, B., \& Solomon, N. G. (2011). avpr1a length polymorphism

661 is not associated with either social or genetic monogamy in free-living prairie voles. Animal

662 Behaviour, 81, 11-18.

663 McGuire, B., \& Getz, L.L. (2010). Alternative male reproductive tactics in a natural population of

664 prairie voles, Microtus ochrogaster. Acta Theriol, 55, 261-270. doi:10.4098/j.at.0001-

$665 \quad 7051.077 .2009$

666 McGuire, B., Getz, L. L., \& Oli, M. K. (2002). Fitness consequences of sociality in prairie voles,

667 Microtus ochrogaster: influence of group size and composition. Animal Behaviour, 64, 645-654.

668 doi:10.1006/anbe.2002.3094.

669 McGuire, B., Pizzuto, T., Getz, L.L. (1990). Potential for social interaction in a natural population

670 of prairie voles (Microtus ochrogaster). Canadian Journal of Zoology, 68, 391-398.

671 Nunn, C.L., Jordán, F., McCabe, C.M., Verdolin, J.L., Fewell, J.H. (2015). Infectious disease and

672 group size: more than just a numbers game. Philosophical Transactions of the Royal Society B,

$673370,20140111$.

674 Okhovat, M., Berrio, A., Wallace, G., Ophir, A. G., \& Phelps, S. M. (2015). Sexual fidelity trade-

675 offs promote regulatory variation in the prairie vole brain. Science, 350, 1371-1374.

676 Ophir, A.G., Wolff, J.O., Phelps, S.M. (2008). Variation in neural V1aR predicts sexual fidelity and

677 space use among male prairie voles in semi-natural settings. PNAS, 105, 1249-1254.

678 Psorakis, I., Roberts, S. J., Rezek, I., \& Sheldon, B. C. (2012). Inferring social network structure in

679 ecological systems from spatio-temporal data streams. Journal of the Royal Society Interface, 9 ,

680 3055-3066. doi: 10.1098/rsif.2012.0223

681 R Core Team (2017). R: A Language and Environment for Statistical Computing. Vienna: R

682 Foundation for Statistical Computing. Available online at: https://www.R-project.org/. 
683 Richmond, M., \& Conaway, C. H. (1969). Management, breeding, and reproductive performance

684 of the vole, Microtus ochrogaster, in a laboratory colony. Laboratory Animal Care, 19, 80-87.

685 Rubenstein, D. R., \& Lovette, I. J. (2007) Temporal environmental variability drives the evolution

686 of cooperative breeding in birds. Current Biology, 17, 1414-1419.

687 doi:10.1016/j.cub.2007.07.032.

688 Ryder, T.B., Parker, P.G., Blake, J.G., \& Loiselle, B.A. (2009). It takes two to tango: reproductive

689 skew and social correlates of male mating success in a lek-breeding bird.

690 Proceedings of the Royal Society B: Biological Sciences, 276, 2377-2384.

691 doi:10.1098/rspb.2009.0208

692 Sabol, A. C., Solomon, N. G., \& Dantzer, B. (2018). How to study socially monogamous behavior

693 in secretive animals? Using social network analyses and automated tracking systems to study

694 the social behavior of prairie voles. Frontiers in Ecology and Evolution, 6, 178.

695 doi:10.3389/fevo.2018.00178

696 Schradin, C., \& Pillay, N. (2005). Intraspecific variation in the spatial and social organization of the

697 African striped mouse, Journal of Mammalogy, 86, 99-107.

698 Schradin, C., König, B., Pillay, N. (2010). Reproductive competition favours solitary living while

699 ecological constraints impose group-living in African striped mice. Journal of Animal Ecology,

$700 \quad 73,515-521$.

701 Schülke, O., Bhagavatula, J., Vigilant, L., \& Ostner, J. (2010). Social bonds enhance reproductive

702 success in male macaques. Current Biology, 20, 2207-2210.

703 doi:10.1016/j.cub.2010.10.058.

704 Shen, S-F., Emlen, S.T., Koenig, W.D., Rubenstein, D.R. (2017). The ecology of cooperative

705 breeding behaviour. Ecology Letters, 20, 708-720.

706 Shuster, S. M., \& Wade, M. J. (2003). Mating systems and mating strategies. Princeton: Princeton

707 University Press. 
708 Shuster, S. M., Willen, R. M., Keane, B., \& Solomon, N. G. (2019). Alternative mating tactics in socially monogamous prairie voles, Microtus ochrogaster. Frontiers in Ecology and Evolution, 7. doi:10.3389/fevo.2019.00007

711 Sikes, R. S., Bryan, J. A., Byman, D., Danielson, B. J., Eggleston, J., Gannon, M. R., Gannon, W.

712 L., Hale, D. W., Jesmer, B. R., Odell, D. K., Olson, L. E., Stevens, R. D., Thompson, T. A.,

713 Timm, R. M., Trewhitt, S. A., \& Willoughby, J. R. (2016). 2016 Guidelines of the American

714 Society of Mammalogists for the use of wild mammals in research and education. Journal of

715 Mammalogy 97, 663-688.

716 Silk, J. B. (2007). The adaptive value of sociality in mammalian groups. Philosophical

717 Transactions of the Royal Society B: Biological Sciences, 362, 539-559.

718 doi:10.1098/rstb.2006.1994

719 Silk, J. B., Alberts, S. C., \& Altmann, J. (2003). Social bonds of female baboons enhance infant

720 survival. Science, 302, 1231-1234. doi:10.1126/science.1088580

721 Silk, J.B., Beehner, J.C., Bergman, T.J., Crockford, C., Engh, A.L., Moscovice, L.R., Wittig, R.M.,

722 Seyfarth, R.M., Cheney, D.L. (2009). The benefits of social capital: close social bonds among

723 female baboons enhance offspring survival. Proceedings of the Royal Society B, 276, 3099-

7243104.

725 Silk, J.B., Beehner, J.C., Bergman, T.J., Crockford, C., Engh, A.L., Moscovice, L.R., Wittig, R.M.,

726 Seyfarth, R.M., Cheney, D.L. (2010). Strong and consistent social bonds enhance the longevity

727 of female baboons. Current Biology, 20, 1359-1361.

728 Solomon, N. G. (1993). Comparison of parental behavior in male and female prairie voles

729 (Microtus ochrogaster). Canadian Journal of Zoology, 71, 1991-1994.

730 Solomon, N. G., \& Keane, B. (2018). Dispatches from the field: sociality and reproductive success

731 in prairie voles. Animal Behaviour, 143, 193-203. doi:10.1016/j.anbehav.2018.07.001

732 Solomon, N. G., \& Jacquot, J. J. (2002). Characteristics of resident and wandering prairie voles,

733 Microtus ochrogaster. Canadian Journal of Zoology, 80, 951-955. 
734 Solomon, N. G., Keane, B., Knoch, L. R., \& Hogan, P. J. (2004). Multiple paternity in socially

735 monogamous prairie voles (Microtus ochrogaster). Canadian Journal of Zoology, 82, 1667-

7361671.

737 Solomon, N.G., Richmond, A. R., Harding, P. A., Fries, A., Jacquemin, S., Schaefer, R. L., Lucia,

738 K. E., \& Keane, B. (2009). Polymorphism at the avpr1a locus in male prairie voles correlated

739 with genetic but not social monogamy in field populations. Molecular Ecology, 18, 4680-4695.

740 Spiegel, O., Sih, A., Leu, S. T., \& Bull, C. M. (2017). Where should we meet? Mapping social

741 network interactions of sleepy lizards shows sex-dependent social network structure. Animal

742 Behaviour, 136, 207-215. doi:10.1016/j.anbehav.2017.11.001.

743 Streatfeild, C. A., Mabry, K. E., Keane, B., Crist, T. O., \& Solomon, N. G. (2011). Intraspecific

744 variability in the social and genetic mating systems of prairie voles, Microtus ochrogaster.

745 Animal Behaviour, 82, 1387-1398. doi: 10.1016/j.anbehav.2011.09.023

746 van Schaik, C. (1983). Why are diurnal primates living in groups? Behaviour, 87, 120-144.

747 Walum, H., \& Young, L. J. (2018). The neural mechanisms and circuitry of the pair bond. Nature

748 Reviews Neuroscience, 19, 643-654. doi:10.1038/s41583-018-0072-6

749 Whitehead, H. (2008). Analyzing animal societies: quantitative methods for vertebrate social

750 analysis. Chicago: University of Chicago Press.

751 Whiteman, N. K., \& Parker, P. G. (2004). Effects of host sociality on ectoparasite population

752 biology. Journal of Parasitology, 90, 939-947. doi:10.1645/GE-310R

753 Wickham, H. (2009). Ggplot2: Elegant graphics for data analysis. New York: Springer-Verlag

754 Young, L. J., \& Wang, Z. (2004). The neurobiology of pair bonding. Nature Neuroscience, 7,

755 1048-1054. doi:10.1038/nn1327

756

757

758

759 
760 Table 1. Effects of the number of social connections on vole mating success (number of

761 different individuals a vole produced offspring with).

\begin{tabular}{|c|c|c|c|c|c|c|}
\hline Social Network & Variable & Estimate & SE & z-value & $\begin{array}{l}\text { P-value } \\
\text { from GLM }\end{array}$ & $\begin{array}{c}\text { P-values from } \\
\text { Randomization Tests }\end{array}$ \\
\hline \multirow[t]{8}{*}{ All individuals } & Intercept & -2.66 & 0.65 & -4.06 & $<0.0001$ & NA \\
\hline & $\operatorname{Sex}(M)$ & 0.68 & 0.92 & 0.73 & 0.46 & NA \\
\hline & Enclosure (LD) & 1.89 & 0.41 & 4.55 & $<0.0001$ & NA \\
\hline & Survival & 2.07 & 0.89 & 2.33 & 0.020 & NA \\
\hline & Degree & 0.083 & 0.21 & 0.40 & 0.69 & $0.12,0.0098,0.14$ \\
\hline & Degree $^{2}$ & -0.012 & 0.013 & -0.88 & 0.38 & $0.012,0.0093,<0.0001$ \\
\hline & Sex $(M) \times$ Degree & -0.21 & 0.23 & -0.90 & 0.37 & $0.021,0.0017,<0.0001$ \\
\hline & Sex (M) $x$ Degree $^{2}$ & 0.014 & 0.015 & 0.94 & 0.35 & $0.01,0.0018,<0.0001$ \\
\hline \multirow[t]{8}{*}{$\begin{array}{l}\text { Opposite-sex } \\
\text { individuals }\end{array}$} & Intercept & -2.81 & 0.70 & -4.08 & $<0.0001$ & NA \\
\hline & $\operatorname{Sex}(M)$ & 0.78 & 0.91 & 0.86 & 0.39 & NA \\
\hline & Enclosure (LD) & 2.03 & 0.43 & 4.72 & $<0.0001$ & NA \\
\hline & Survival & 1.75 & 0.84 & 2.09 & 0.037 & NA \\
\hline & Degree & 0.19 & 0.31 & 0.63 & 0.53 & $0.18,0.19,<0.0001$ \\
\hline & Degree $^{2}$ & -0.028 & 0.028 & -1.01 & 0.31 & $0.0011,0.0035,0.001$ \\
\hline & Sex $(M) \times$ Degree & -0.53 & 0.38 & -1.42 & 0.15 & $<0.0001,<0.0001,0.0046$ \\
\hline & Sex (M) $x$ Degree $^{2}$ & 0.057 & 0.042 & 1.36 & 0.17 & $<0.0001,<0.0001,0.0068$ \\
\hline
\end{tabular}

762 Degree refers to the number of social connections for each individual either with all voles or

763 opposite-sex voles. Note that relationships involving social network data have three $P$-values

764 because those regression coefficients were compared to those from randomized networks three

765 times to determine if they were consistently significant (see methods for details). Survival refers to

766 the proportion of days the vole was in the enclosure based on when it was last recorded. "LD" is

767 low-density enclosure.

768 
Table 2. Effects of the number of social connections on vole reproductive success (number of

770 offspring produced that survived until emergence from the natal nest).

\begin{tabular}{|c|c|c|c|c|c|c|}
\hline Social Network & Variable & Estimate & SE & z-value & $\begin{array}{l}\text { P-value } \\
\text { from GLM }\end{array}$ & $\begin{array}{c}\text { P-values from } \\
\text { Randomization Tests }\end{array}$ \\
\hline \multirow[t]{8}{*}{ All individuals } & Intercept & -2.90 & 0.66 & -4.14 & $<0.0001$ & NA \\
\hline & $\operatorname{Sex}(M)$ & 0.61 & 0.93 & 0.65 & 0.52 & NA \\
\hline & Enclosure (LD) & 2.10 & 0.40 & 5.24 & $<0.0001$ & NA \\
\hline & Survival & 2.49 & 0.82 & 3.03 & 0.0025 & NA \\
\hline & Degree & 0.053 & 0.19 & 0.28 & 0.78 & $0.33,0.25,0.19$ \\
\hline & Degree $^{2}$ & -0.0085 & 0.011 & -0.77 & 0.44 & $0.002,0.035,0.015$ \\
\hline & Sex $(M) \times$ Degree & -0.14 & 0.21 & -0.64 & 0.52 & $0.12,0.18,0.12$ \\
\hline & Sex $(M) \times$ Degree $^{2}$ & 0.0082 & 0.013 & 0.63 & 0.53 & $0.021,0.11,0.022$ \\
\hline \multirow[t]{8}{*}{$\begin{array}{l}\text { Opposite-sex } \\
\text { individuals }\end{array}$} & Intercept & -2.97 & 0.67 & -4.41 & $<0.0001$ & NA \\
\hline & $\operatorname{Sex}(M)$ & 0.58 & 0.91 & 0.64 & 0.53 & NA \\
\hline & Enclosure (LD) & 2.21 & 0.41 & 5.40 & $<0.0001$ & NA \\
\hline & Survival & 2.19 & 0.77 & 2.83 & 0.0046 & NA \\
\hline & Degree & 0.15 & 0.28 & 0.52 & 0.60 & $0.20,0.53,0.54$ \\
\hline & Degree $^{2}$ & -0.022 & 0.024 & -0.93 & 0.35 & $0.14,0.099,0.12$ \\
\hline & Sex $(M) \times$ Degree & -0.39 & 0.38 & -1.04 & 0.30 & $0.047,0.024,0.11$ \\
\hline & Sex $(M) \times$ Degree $^{2}$ & 0.038 & 0.041 & 0.92 & 0.36 & $0.063,0.010,0.02$ \\
\hline
\end{tabular}

771 Degree refers to the number of social connections for each individual either with all voles or

772 opposite-sex voles. Note that relationships involving social network data have three $P$-values

773 because those regression coefficients were compared to those from randomized networks three

774 times to determine if they were consistently significant (see methods for details). Survival refers

775 to the proportion of days the vole was in the enclosure based on when it was last recorded. "LD"

776 is low-density enclosure. 
777 Table 3. Overall effects of vole mating success (number of different individuals with which a

778 vole produced offspring) on reproductive success (number of offspring produced that survived

779 until emergence from the natal nest).

\begin{tabular}{lcccc}
\hline \multicolumn{1}{c}{ Variable } & Estimate & SE & z-value & $\begin{array}{c}\text { P-value } \\
\text { from GLM }\end{array}$ \\
\hline Intercept & $\mathbf{- 2 . 1 3}$ & $\mathbf{0 . 4 4}$ & -4.83 & $<\mathbf{0 . 0 0 0 1}$ \\
Number of Mates & $\mathbf{0 . 6 4}$ & $\mathbf{0 . 1 3}$ & $\mathbf{4 . 8 8}$ & $<\mathbf{0 . 0 0 0 1}$ \\
Sex (M) & 0.26 & 0.49 & 0.53 & 0.60 \\
Enclosure (LD) & $\mathbf{1 . 5 3}$ & $\mathbf{0 . 4 5}$ & $\mathbf{3 . 3 7}$ & $\mathbf{0 . 0 0 0 7 6}$ \\
\# of Mates x Sex (M) & -0.093 & 0.17 & -0.56 & 0.58
\end{tabular}

780 Since this model did not include social network data we did not perform the randomizations to

781 generate three $P$-values, we present the $P$-values from the GLM. "LD" is low-density enclosure. 
783 Table 4. Effects of the number of social connections on average body mass for males.

\begin{tabular}{|c|c|c|c|c|c|c|}
\hline Social Network & Variable & Estimate & SE & t-value & $\begin{array}{l}\text { P-value } \\
\text { from LM }\end{array}$ & $\begin{array}{c}\text { P-values from } \\
\text { Randomization Tests }\end{array}$ \\
\hline \multirow[t]{6}{*}{ All individuals } & Intercept & 43.42 & 2.70 & 16.10 & $<0.0001$ & NA \\
\hline & Enclosure (LD) & -1.03 & 2.57 & -0.40 & 0.69 & NA \\
\hline & Survival & 13.37 & 8.78 & 1.52 & 0.14 & NA \\
\hline & \# of measures & -0.12 & 0.60 & -0.20 & 0.84 & NA \\
\hline & Degree & 0.95 & 0.56 & 1.70 & 0.099 & $<0.0001,0.0001,<0.0001$ \\
\hline & Degree $^{2}$ & -0.066 & 0.028 & -2.36 & 0.025 & $<0.0001,<0.0001,<0.0001$ \\
\hline \multirow[t]{6}{*}{$\begin{array}{l}\text { Opposite-sex } \\
\text { individuals }\end{array}$} & Intercept & 44.31 & 2.81 & 15.78 & $<0.0001$ & NA \\
\hline & Enclosure (LD) & -0.16 & 2.80 & -0.058 & 0.95 & NA \\
\hline & Survival & 10.80 & 8.89 & 1.22 & 0.23 & NA \\
\hline & \# of measures & -0.20 & 0.63 & -0.31 & 0.76 & NA \\
\hline & Degree & 1.54 & 1.22 & 1.26 & 0.22 & $0.0035,0.0036,0.0332$ \\
\hline & Degree $^{2}$ & -0.20 & 0.13 & -1.54 & 0.13 & $0.0024,0.0031,0.0005$ \\
\hline
\end{tabular}

784 Degree refers to the number of social connections for each individual either with all voles or

785 opposite-sex voles. Relationships involving social network data have three $P$-values because

786 those regression coefficients were compared to those from randomized networks three times to

787 determine if they were consistently significant (see methods). "\# of measures" refers to number

788 of times we measured body mass, which were used to generate average mass for each

789 individual. Survival refers to proportion of days the vole was in the enclosure. "LD" is low-density

790 enclosure.

791

792

793

794

795 
796 Figure 1. a) The number of females, males, and offspring in each of our two enclosures over

797 time during the study based on the number of unique individuals live-trapped during each two-

798 week period of the field season. Note that the area of enclosures is equal, so the number of

799 voles in each enclosure can be used to compare relative density between the two, b) The sex

800 ratio in each enclosure, calculated as the proportion of total adult voles trapped during each

801 two-week period of the field season that were males, over time of the study.
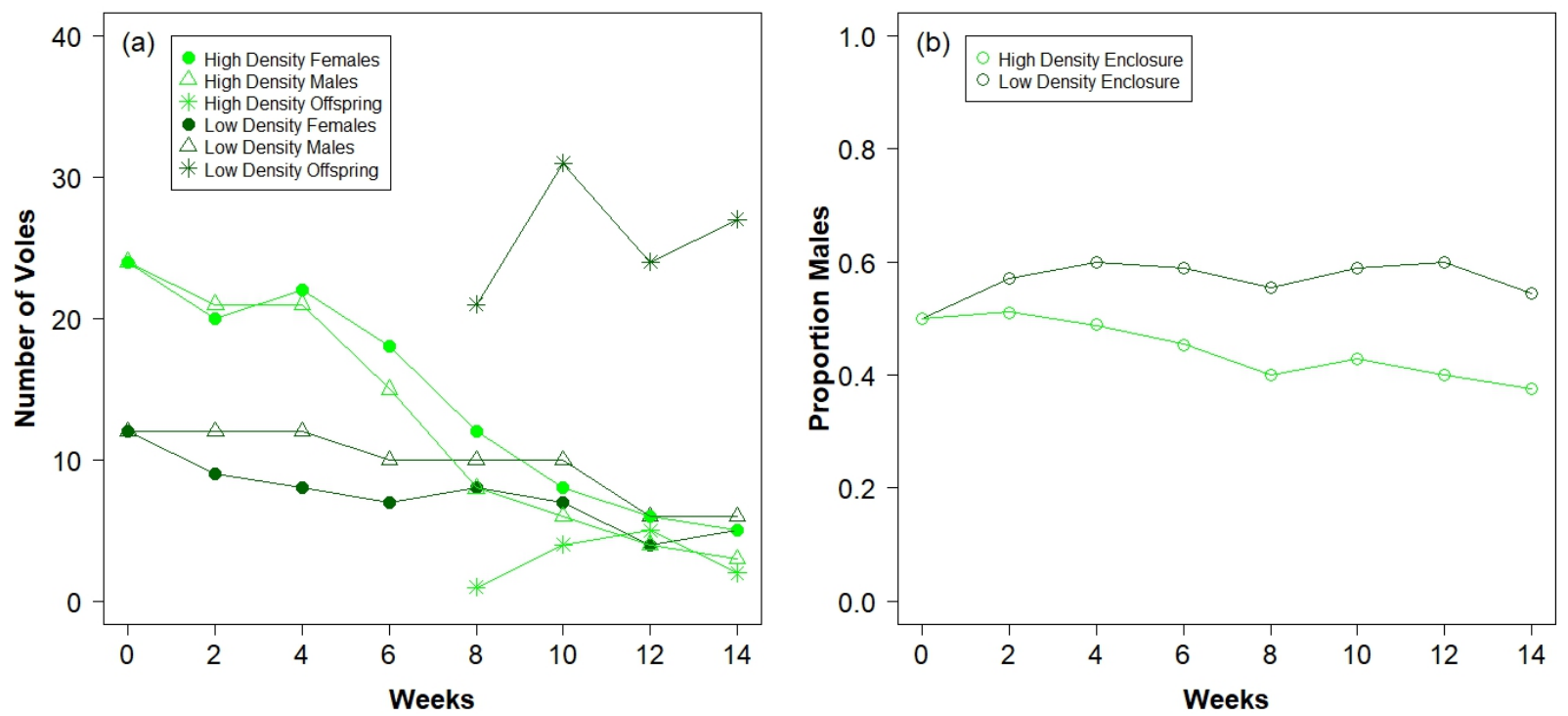
804 Figure 2. Both females and males with an intermediate number of social connections with a) all

805 voles in their enclosure (both same- or opposite-sex individuals) or b) only opposite-sex

806 individuals in their enclosure had higher mating success (defined as the number of different

807 individuals with which they produced offspring). Points are jittered with males shown in blue and

808 females in red. Full statistical results shown in Table 1.
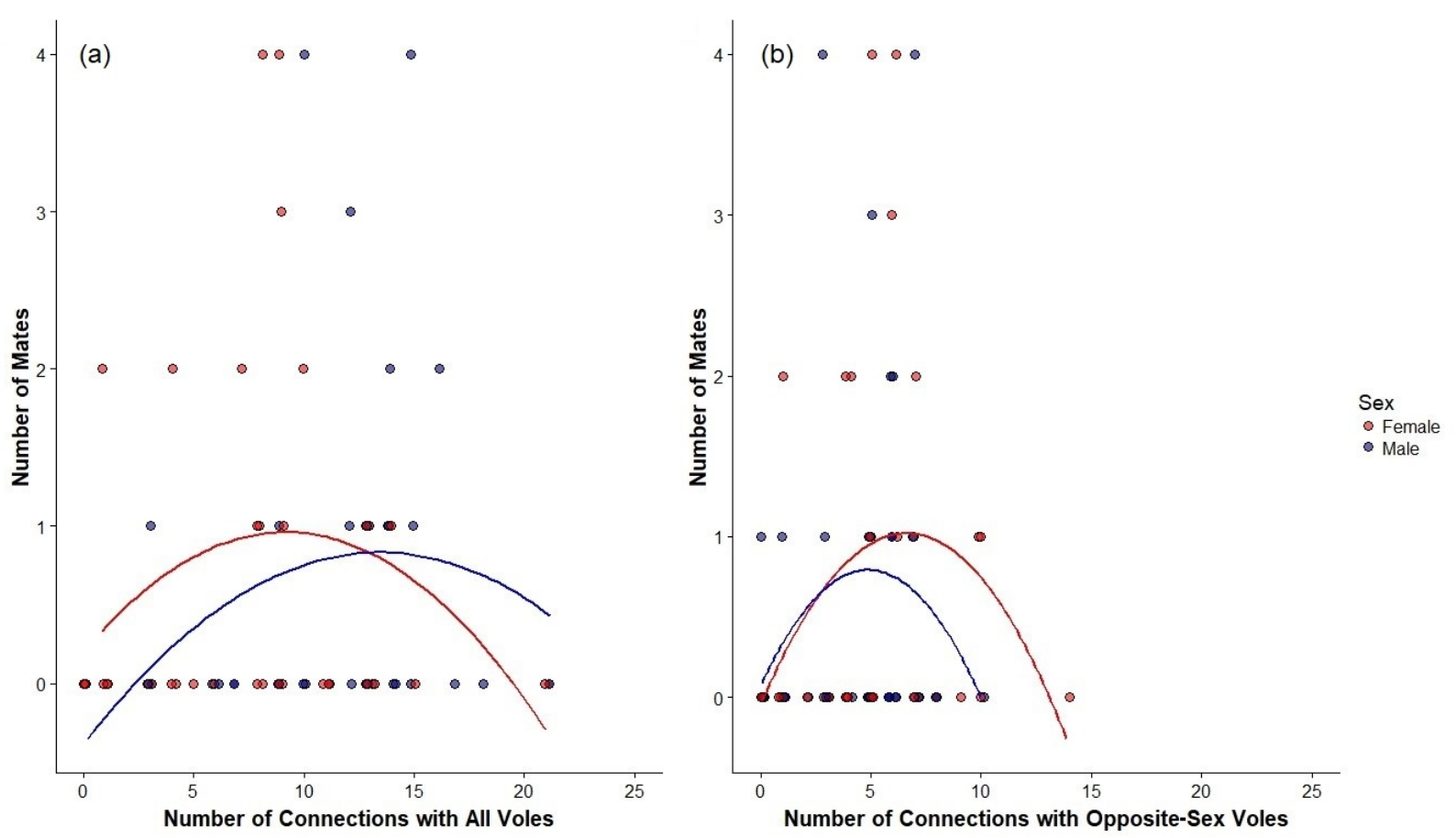
811 Figure 3. a) Both females and males with an intermediate number of social connections with all

812 voles in their enclosure (both same- and opposite-sex individuals) produced more offspring that

813 survived to emergence from the natal nest. b) Female and male voles with an intermediate

814 number of social connections with only opposite-sex individuals in their enclosure also tended to

815 produce more offspring. Points are jittered with red points being females and blue points being

816 males. Full statistical results shown in Table 2.
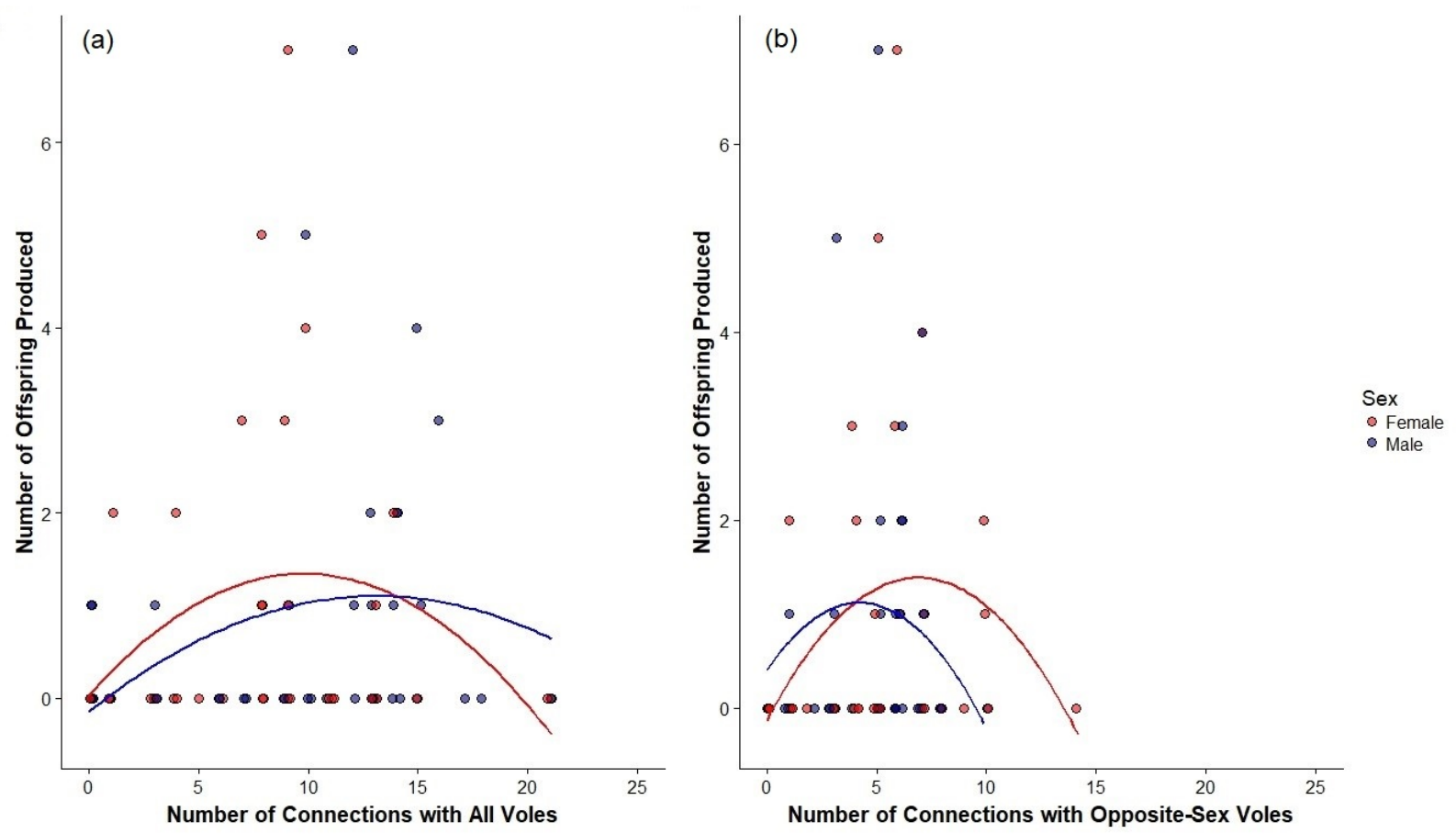

818 
819 Figure 4. Both female and male prairie voles that had higher mating success (produced

820 offspring with a greater number of different mates) produced a great number of offspring that

821 survived to emergence from the natal nest. Points for females and males are jittered. Full results

822 shown in Table 3.

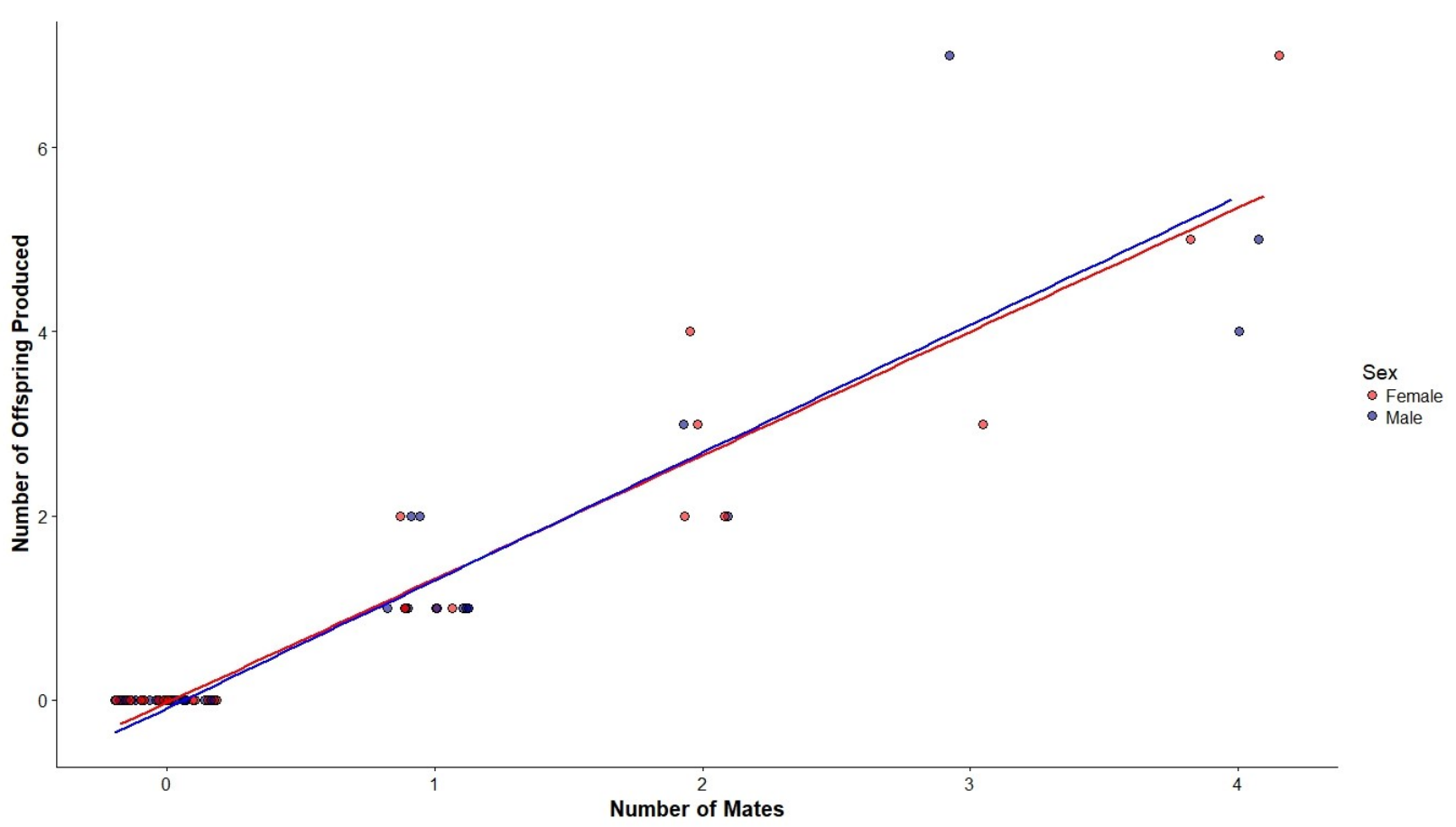


825 Figure 5. Male voles with an intermediate number of social connections a) with both female and

826 male voles in their enclosure or b) with just female voles, were significantly heavier over the

827 course of this study. Body mass for males was averaged for the entire duration of this study.

828 The number of times we measured body mass ("N") varied among males so the size of each

829 point is scaled based on the number of recorded mass measurements we have for each

830 individual. Full results shown in Table 4.
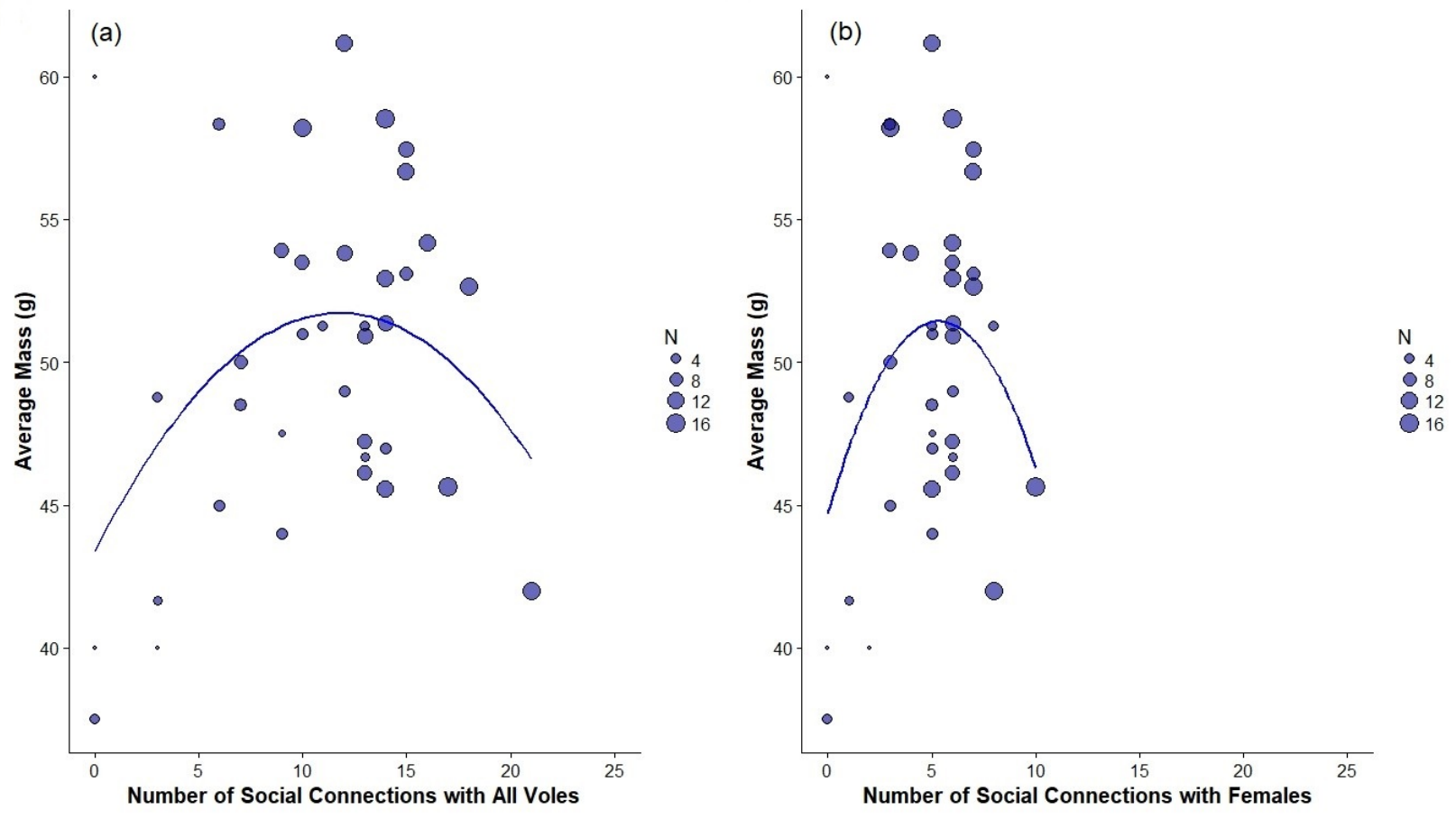

832 
833 Figure A1. Layout of the two RFID arrays in the enclosures. The RFID system was kept at each

834 array in each enclosure for three days in the order: array 1 enclosure 1, array 2 enclosure 1,

835 array 1 enclosure 2, and array 2 enclosure 2 and then repeated for the duration of the field

836 season.

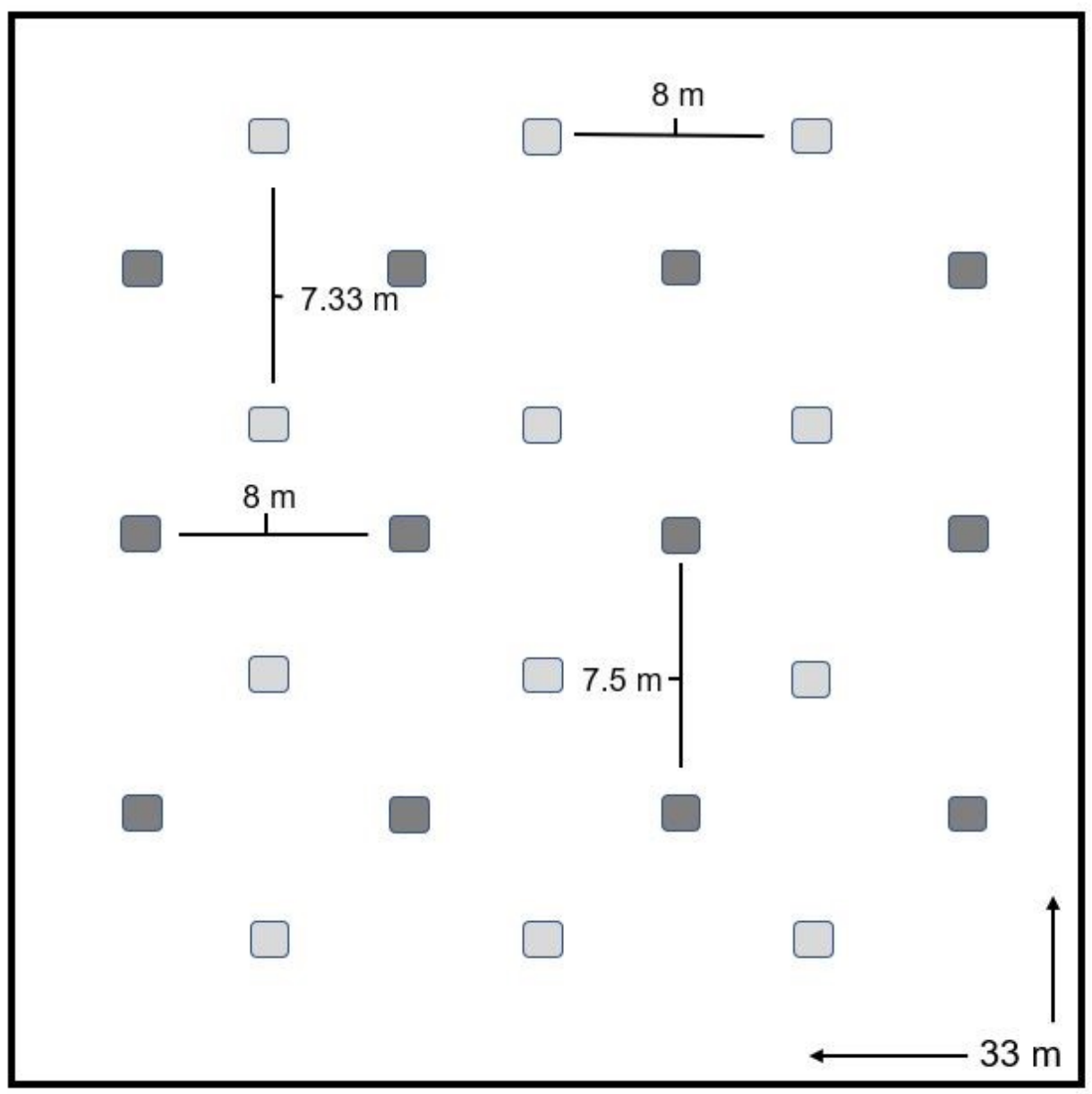

